\title{
Archéologie du Moyen Âge
}

\section{Édith Peytremann}

\section{(2) OpenEdition}

\section{Journals}

Édition électronique

URL : http://journals.openedition.org/rao/3186

DOI : 10.4000/rao.3186

ISBN : 978-2-7535-5014-8

ISSN : 1775-3732

\section{Éditeur}

Presses universitaires de Rennes

Édition imprimée

Date de publication : 31 décembre 2015

ISBN : 978-2-7535-5012-4

ISSN : 0767-709X

\section{Référence électronique}

Édith Peytremann, « Archéologie du Moyen Âge », Revue archéologique de l'Ouest [En ligne], 32 | 2015, mis en ligne le 28 avril 2016, consulté le 02 mars 2021. URL : http://journals.openedition.org/rao/3186 ; DOI : https://doi.org/10.4000/rao.3186 
années 2000, en centre d'interprétation dédié à l'architecture romane. C'est aussi dans ce cadre que le cloître a fait l'objet de plusieurs fouilles programmées de 2001 à 2005, dont les résultats forment l'essentiel de l'article. Les apports de cette fouille sont divers. Outre la mise en évidence de légères variations dans l'orientation des bâtiments, la fouille a également livré des sépultures attestant de modes d'inhumations diversifiés et de beaux ensembles mobiliers, partiellement illustrés dans l'article.

L'ouvrage s'achève sur une approche patrimoniale des bâtiments monastiques, dont le statut et la perception changent selon la période considérée, avec la désacralisation brutale des lieux à la Révolution, entrainnant de nouvelles affections jusqu’à leur réutilisation récente comme espace culturel. C'est là une ouverture originale qui est offerte au lecteur sous la forme de quatre contributions réunies dans une partie intitulée " Politiques patrimoniales" (p. 249-293).

La première correspond à un chapitre de synthèse consacré au patrimoine architectural monastique en PoitouCharentes. Véronique Dujardin y retrace « les processus et des étapes de protection au titre des monuments historiques " du $\mathrm{XIX}^{\mathrm{e}}$ siècle à nos jours. Les contributions suivantes concernent la mise en valeur de trois établissements différents que sont le centre d'interprétation de l'architecture romane de SaintAmant-de-Boixe (Charente) présenté par Marylise Ortiz et Anaël Vignet, l'abbaye de Fontevraud (Maine-et-Loire) par Daniel Prigent et l'abbaye Saint-Vincent-de-Nieul-surl'Autise (Vendée) par Richard Levesque.

L'ouvrage, auquel il manque peut-être une conclusion générale, s’achève sur une bibliographie régionale abondante (p. 295-307) et un appréciable index des noms de lieux (p. 309-313).

L'objectif de départ, qui était d'alterner synthèses et études de cas, n'est pas tout à fait atteint puisque l'ouvrage, ne comprend au final, que trois ou quatre approches territoriales sur la vingtaine de contributions recensées. Ce sont ici les études monographiques qui sont privilégiées et qui présentent souvent des données inédites, par exemple sur l'origine des établissements monastiques, notamment lorsqu'elles résultent d'opérations archéologiques récentes. On retiendra par ailleurs l'apport des études de bâti dans la connaissance de plusieurs bâtiments.

D'un point de vue formel, on peut regretter que les illustrations soient parfois difficilement lisibles, les cartes de synthèses et un certain nombre de plans étant présentés dans le corps du texte à une échelle trop réduite. Ce défaut est partiellement atténué par la présentation des mêmes documents, en couleur et à une échelle plus adaptée, dans les planches du cahier central.

Quoi qu'il en soit, on ne peut que saluer la parution de cet ouvrage qui couronne un travail collectif rondement mené et qui offre un bel état des connaissances sur un territoire donné. Puisse-t-il susciter de nouveaux travaux de recherche et être une source d'inspiration pour d'autres espaces régionaux.

Yves Henigfeld (Université de Nantes)

Burnouf Joëlle et Catteddu Isabelle, 2015 - Archéologie du Moyen Âge, Rennes, éditions Ouest-France et Inrap, 143 p. (ISBN 978-2-7373-6701-4).

L’ouvrage réalisé par Joëlle Burnouf et Isabelle Catteddu, publié aux éditions Ouest-France/Inrap s'adresse à un large public auquel les deux auteures veulent faire passer un message, le Moyen Âge ce n'est pas ce que vous croyez! et proposer la découverte d'un autre Moyen Âge en s'appuyant principalement sur les résultats des fouilles archéologiques de ces trente dernières années.

Pour ce faire, les auteures débutent leur ouvrage de manière originale en invitant le lecteur à s'interroger sur le temps qu'il faisait à l'époque et comment était l'environnement. Cette partie particulièrement didactique augure bien des renouveaux de la recherche climatique grâce aux apports des nombreuses fouilles archéologiques et des travaux interdisciplinaires qu' elles suscitent.
Ainsi, après avoir dressé rapidement à l'aide de quelques exemples un état des lieux en quatre étapes des changements climatiques et de leurs répercussions sur l'environnement et sur les sociétés, après avoir présenté les paysages déjà fortement aménagés depuis, au moins l'âge du Bronze et montré que l'interaction hommes-milieux fabrique une dynamique créatrice de changements, les auteures font pénétrer le lecteur dans les campagnes médiévales. Elles suivent alors une démarche chronologique pour, tour à tour, exposer l'agriculture et ses développements au cours du millénaire avec notamment l'introduction de nouvel outillage, le développement de l'élevage ou plus tard celui du maraîchage ou de la fruiticulture; les manières d'habiter avec ces différents schémas d'occupation des sols et sa diversité 
des constructions pouvant, pour certaines, matérialiser des lieux de pouvoir. Là encore des exemples simples viennent illustrer les propos des auteures.

Le lecteur est ensuite conduit en ville où toujours selon un schéma chronologique, on tente de lui montrer les développements urbains du premier Moyen Âge où la ville est principalement qualifiée de " chrétienne » jusqu’au second Moyen Âge où s'invente la ville moderne. Tout comme pour le chapitre précédent, les activités économiques comme les lieux d'habitat ou de travail sont décrits simplement.

À présent que le lecteur est plus familiarisé avec la période, les auteures attirent cette fois-ci son attention sur l'ingéniosité dont il est fait preuve au Moyen Âge dans un chapitre carrément intitulé " une première révolution industrielle »! De manière dynamique, un certain nombre d'innovations médiévales sont déclinées dans les domaines aussi variées que l'agriculture, les artisanats des matières animales, les arts du feu, l'exploitation des matières premières et leur transformation. Ce chapitre, à la suite du premier, attire l'attention sur la gestion de l'environnement et l'épuisement aussi bien des sols, que des espèces végétales et animales.

Mais qui sont donc ces femmes et ces hommes du Moyen Âge? C'est dans un chapitre consacré à " une société multiculturelle et métissée " que les auteurs présentent aussi bien les pratiques funéraires et ce qu'elles enseignent sur les sociétés, le niveau de santé des populations en évoquant notamment le handicap et son traitement au Moyen Âge à l'aide de quelques exemples archéologiques, que la diversité d'origine des populations médiévales métropolitaines et leur intégration.

Pour achever ce parcours initiatique du Moyen Âge, les auteurs invitent allègrement le lecteur à bouger en empruntant un réseau viaire beaucoup plus important que le laisse entendre la tradition, en montant sur des embarcations qui naviguent aussi bien sur les cours d'eau, les littoraux ou en haute mer, en s'arrêtant dans des ports, en rejoignant des foires, en suivant les moines, les pèlerins ou les marchands.

Lorsque le lecteur ferme le livre, il a du mal à quitter le dynamisme médiéval qui lui a été donné de voir! Le pari des auteures de présenter un autre Moyen Âge est à coup sur gagné. L’obscurité dont était affublée cette période de l'histoire disparaît au profit d'une lumière, un peu trop brillante peut-être, qui montre une société adaptable, inventive, intégratrice. De l'aveu même des auteures, certains aspects ont volontairement été délaissés. Si la collection ne permet en effet pas de tout traiter, l'évocation de certains aspects de la société médiévale (guerre, église) auraient probablement permis de contrebalancer ce tableau du Moyen Âge qui peut parfois apparaitre du coup un peu surexposé. Soulignons avec force la qualité de la présentation didactique des résultats les plus récents de la recherche avec de réelles originalités. L'ensemble du propos est appuyé, toujours fort à propos, par une illustration de choix et de grande qualité qui contribuent grandement à celle de l'ouvrage. Il est par ailleurs plaisant d'y trouver un certain renouvellement par rapport aux ouvrages du même éditeur.

Les professionnels trouveront par-ci, par-là des approximations, des développements un peu vagues notamment sur la ville du premier Moyen Âge, qu'importe. Il se trouve là un ouvrage, pas trop cher, qui permet à tous de pouvoir se faire une idée relativement précise, à l'aide des résultats les plus récents de la recherche archéologique, d'une longue période, loin des sempiternels clichés qui malheureusement sont toujours à l'honneur des animations culturelles estivales et des parcs d'attractions. On ne peut, ici, que saluer les auteures qui ont toujours réussi à exposer et démontrer les faits, sans concession, pour le savoir du plus grand nombre.

Édith Peytremann (Inrap, UMR 6273)

Tendron G., 2014 - Yzeures-sur-Creuse (37). Les monuments romains, Chauvigny, Association des Publications Chauvinoises, coll. «Memoria Momenti; $32 », 95$ p. (ISBN 979-10-90534-24-7).

Dans cet opuscule richement illustré, Graziella Tendron rend compte de l'étude qu'elle a consacrée à des fragments d'architecture romaine découverts à la fin du XIX ${ }^{e}$ siècle, lors d'une réfection de l'église médiévale d'Yzeures-sur-Creuse. Leur découverte attira le révérend père jésuite Camille de la Croix, fondateur de l'archéologie poitevine, qui organisa une campagne de fouille en février 1896 permettant d'extraire entre 85 et 95 blocs. 52 d'entre eux sont conservés au sein du musée Minerve du bourg et ont fait l'objet d'une nouvelle présentation en 2014, donnant l'occasion de les réexaminer.

Ces membra disjecta correspondent en fait, si l'on excepte quelques morceaux de sarcophages du haut Moyen Âge, à des portions de monuments relevant d'un sanctuaire antique qui, après son abandon, ont été récupérés pour être remployés, très vraisemblablement, au sein d'une église mérovingienne antérieure à l'actuelle. 21 blocs permettent 